\title{
Avaliação do perfil epidemiológico das crianças internadas no setor de oncologia pediátrica do Hospital da Criança em Feira de Santana.
}

\section{$\underline{\text { Ágda Braga Teixeira }}^{1}$; Marcio Campos Oliveira ${ }^{2}$; Brisa Macêdo Costa ${ }^{3}$ Maria da}

Conceição Andrade ${ }^{4}$

1. Bolsista PIBIC/CNPq, Graduando no curso de Odontologia, Universidade Estadual de Feira de Santana, e-mail: agda_braga@hotmail.com

2. Professor doutor, Departamento de saúde, Universidade Estadual de Feira de Santana, e-mail: marciopatologiaoral@gmail.com

3. Bolsista Fapesb, Departamento de saúde, graduanda no curso de medicina, Universidade Estadual de Feira de Santana, e-mail: brisa.xp@hotmail.com

4. Professora Doutora, Departamento de saúde, Universidade Estadual de Feira de Santana, e-mail: conceicao.icb@gmail.com

PALAVRAS-CHAVE: Leucemias, linfomas, incidência, perfil epidemiológico.

INTRODUÇÃO: O câncer faz parte do grupo de doenças crônico-degenerativas que vem ocorrendo em larga escala na população. Podem originar-se em todo e qualquer tecido humano e em qualquer idade, alterando o perfil de morbimortalidade da população brasileira (CARGNIN; LISTON, 2004). Diferentemente do câncer em adultos, o câncer infantil é raro. A incidência de câncer em crianças é baixa, quando comparada com outras doenças pediátricas. A taxa de incidência do câncer infantil tem crescido em torno de 1\% ao ano (RODRIGUÊS; CAMARGO, 2003). Fato este que leva a atrasos no diagnóstico, tornando o câncer uma das principais causas de óbito em pediatria e de perda potencial de anos de vida (SANTANA et al, 2006).

No entanto, as neoplasias da infância merecem atenção especial, em virtude do desgaste psíquico e social que acarretam, assim como dos elevados custos financeiros envolvidos no diagnóstico, no tratamento e no atendimento às sequelas (DINIZ et al, 2005).

Uma compreensão mais afinada dos aspectos psicológicos, ambientais, genéticos e sociais que envolvem a criança com câncer e sua família, respeitando suas individualidades e especificidades, enfim, toda a complexidade desse ser em crescimento inserido em um meio sócio-cultural-religioso contribui para uma assistência integral a estes pacientes. O presente trabalho tem como objetivo descrever aspectos epidemiológicos como tipos de câncer, aspectos demográficos e possíveis fatores de risco (genéticos e ambientais) e relações familiares, trazendo as representações sociais pertencentes ao núcleo doença em pacientes atendidos em uma unidade de tratamento de câncer infantil.

MATERIAL E MÉTODOS: A pesquisa realizada é do tipo Estudo epidemiológico de corte transversal. A população estudada foi constituída por crianças internadas no setor de oncologia do Hospital da Criança em Feira de Santana -Ba, no período de setembro de 2016 a agosto de 2017. Sendo eleitas ao estudo crianças que possuíam diagnóstico de câncer, idade 
até 18 anos; estando em fase de tratamento ou já tê-lo concluído na instituição supracitada; sendo excluídos os pacientes com tumores benignos e doenças hematológicas, que também são atendidos no local; que abandonaram o tratamento. Após o recrutamento inicial dos pacientes elegíveis ao estudo, os mesmos responderam a um questionário constituído de perguntas relacionadas às características sociodemográficas, de hábitos de vida e história médica-odontológica. Todas as informações foram registradas em uma ficha de acompanhamento, a qual permitiu posterior avaliação de possíveis alterações dessas variáveis.

RESULTADOS E/OU DISCUSSÃO: De acordo com a literatura, a incidência total de tumores malignos na infância geralmente é maior em crianças do gênero masculino (PERES et al, 2013), com uma proporção de 1:1 a 1:4, o que pode indicar uma fragilidade constitucional no gênero masculino ou uma associação com fatores genéticos de predisposição ligados ao cromossomo X (MILLER; RIES; HANKEY, 1993), porém, no estudo em questão, a maior parte dos pacientes atendidos na instituição era do gênero feminino.

A maioria das crianças encontrava-se na faixa etária de 0 a 5 anos. Para Peres et al (2013), com relação à faixa etária, a incidência do câncer infantil é maior no primeiro ano de vida, apresentando um segundo pico entre o segundo e terceiro anos de vida. Petrilli e colaboradores (1997) relatam ainda que essa é a faixa mais acometida com exceção de linfomas, carcinomas e tumores ósseos, que predominam em crianças entre 10 e 14 anos.

Na população estudada, as leucemias foram o tipo de câncer mais frequente, representando $54,7 \%$ dos casos no gênero masculino e $49,9 \%$ dos casos no feminino. Segundo Camargo (2008), na faixa etária compreendida entre 0 e 14 anos, a leucemia linfocítica aguda (LLA) foi o tumor mais comum, correspondendo a $23 \%$ dos cânceres. A leucemia não-linfóide ou mielóide aguda foi o segundo tipo mais comum, ocorrendo numa taxa de 1:5 da LLA. Os tumores do sistema nervoso central (SNC) corresponderam a $22,1 \%$ e em conjunto com ambas as leucemias corresponderam à metade de todos os cânceres que ocorreram nessa faixa etária. Os outros tipos de tumores sólidos mais comuns foram o neuroblastoma $(7,7 \%)$, seguido do tumor de Wilms (5,9\%). Em crianças, a leucemia linfocítica aguda (LLA) é a forma de leucemia mais comum, totalizando 75 a $80 \%$ de todas as leucemias (SHARP; COTTON; LITTLE, 1999).

Os Linfomas foram o segundo grupo mais diagnosticado, seguidos pelos tumores de cérebro, o que está em conformidade com a literatura mundial. Esse grupo de neoplasias corresponde às categorias: Il.a. Doença de Hodgkin (DH); Il.b. Linfomas não-Hodgkin (LNH); II.c. Linfoma de Burkitt; II.d. Miscelâneas de neoplasias linfo-reticulares e II.e Linfomas não especificados. Correspondem ao terceiro tipo de câncer mais comum em crianças norte americanas, após as 
leucemias e os tumores do sistema nervoso central (LITTLE, 1999). Já nos países em desenvolvimento correspondem ao segundo lugar, ficando atrás apenas das leucemias (BRAGA, 2002).

Quanto a renda familiar e grau de escolaridade dos pais dos participantes da pesquisa, percebeu-se que em sua maioria, os participantes exibem um nível socioeconômico baixo, o que pode ser explicado também pelo fato da pesquisa ter sido realizada em uma instituição pública.

Embora o câncer seja a causa de morte por doença mais comum em crianças no mundo desenvolvido, com a melhoria da sobrevida, a taxa de mortalidade reduziu em 30 por milhão de crianças ao ano. Diferentemente do que ocorre com a população adulta, onde a prevenção primária e o diagnóstico precoce são as ferramentas mais importantes contra o câncer, na infância e adolescência a terapêutica tornou-se a principal estratégia contra a doença. $A$ sobrevida das crianças e adolescentes com doenças malignas melhorou sensivelmente nos últimos 30 anos e isto se deve, sobretudo, à melhoria e à centralização do tratamento em instituições especializadas (AMADO, 2013). Exames por imagem, que permitem diagnóstico mais precoce e preciso, ou a utilização de técnicas de biologia molecular, que detectam mutações cromossômicas e outras anormalidades genéticas, favorecem uma utilização mais eficiente dos métodos terapêuticos empregados - quimioterapia, cirurgia e radioterapia propiciando um aumento nas taxas de sobrevida, uma melhoria na qualidade de vida e um decréscimo nas taxas de mortalidade (LATORRE; FRANCO, 1996).

CONSIDERAÇÕES FINAIS: A etiologia do câncer infantil ainda não está totalmente elucidada, desta forma, o entendimento dos fatores sociais, genéticos, econômicos e demográficos onde estão inseridos os indivíduos acometidos pela doença parecem ser de suma importância do ponto de vista de se buscar medidas de prevenção e tratamento cada vez mais efetivas, sendo assim, trabalhos similares precisam ser realizados, com vistas ao embasamento da literatura sobre a temática. Em nossa pesquisa, leucemias e linfomas foram as neoplasias malignas mais prevalentes nos pacientes de nossa amostra, seguidos dos tumores cerebrais, conforme a literatura, no entanto, o gênero feminino foi o mais acometido, além de que a doença acometeu mais indivíduos de classe socioeconômica baixa e com pais exibindo, predominantemente, baixos níveis de escolaridade. 


\section{REFERÊNCIAS:}

1.CARGNIN, E. R. G; LISTON N. M; DUPAS, G. Representação social da criança sobre o cancer. Rev Esc Enferm USP 2004.

2.RODRIGUES, K. E; CAMARGO, B. DIAGNÓSTICO PRECOCE DO CÂNCER INFANTIL: RESPONSABILIDADE DE TODOS. Rev Assoc Med Bras 2003;

3.SANTANA, L. R; et al Perfil Epidemiológico das Leucemias em Crianças e Adolescentes no Estado da Bahia. Gazeta Médica da Bahia. 2006.

4.DINIZ, A. B. et al. Perfil epidemiológico do câncer infantil em população atendida por uma unidade de oncologia pediátrica em Salvador-Bahia. R. Ci. méd. biol., Salvador, v. 4, n. 2, p. 131-139, mai./ago. 2005

5. PERES. P. et al. Odontopediatria aplicada ao câncer infantil - manifestações 6.clínicas e protocolos de atendimento, J Manag Prim Health Care, 2013.

7. MILLER, B.A .; RIES, L.A.G .; HANKEY, B.F. SEER revisão das estatísticas do câncer 1973-1990. Bethesda:National Cancer Institute, 1993. (NIH pub 93-27898. PETRILLI, A.S. et al. Diferenças clínicas, epidemiológicas e biológicas entre o câncer na criança e no adulto. R. Bras. Cancerol., Rio de Janeiro, v.43, n.3, p.191-203, 1997.

9. CARNEIRO. F. M.; SILVA. L. C. P.; CRUZ. R. A. Manifestações bucais das leucemias agudas na infância, Arq bras odontol, Minas Gerais, 2008.

10. SHARP, L.; COTTON, A.; LITTLE, J. Descriptive epidemiology. In: LITTLE, J. (Ed.) Epidemiology of childhood cancer. Lyon: International Agency for Research on Cancer, 1999. p.10-16 (IARC Scientific Publications 149).

11. BRAGA, P.E.; LATORRE; M.R.D.O; CURADO, M.P. Câncer na infância: análise comparativa da incidência, mortalidade e sobrevida em Goiânia(Brasil) e outros países. Cad. Saúde Pública, Rio de Janeiro, v.18, n.1, p.33-44, 2002.

12. AMADO. C. F. Câncer na infância e adolescência: caracterização epidemiológica a partir do relacionamento do registro de câncer de base populacional e do sistema de informação de mortalidade, Centro de Ciências da Saúde da Universidade Federal de Pernambuco, Recife, 2013. 